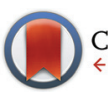

CrossMark

Cite this: Polym. Chem., 2015, 6, 426

\title{
Synthesis of polymeric janus nanoparticles and their application in surfactant-free emulsion polymerizations
}

\author{
Binh T. T. Pham, ${ }^{a}$ Chris H. Such ${ }^{b}$ and Brian S. Hawkett*a
}

A robust and simple synthesis of nano-size oblate to dumbbell shaped polymeric anisotropic particles using RAFT mediated emulsion polymerization is presented. The particle synthesis relies on the property that monomer swollen cross-linked polymer seed particles shrink and expel some of the monomer when heated. Thus, upon heating for polymerization, some of the swelling monomer is expelled and subsequently polymerizes to form a bulge on the side of the original crosslinked seed particle. The shape of the bulge, and the degree of contact that the expelled monomer maintains with the original seed particle, is controlled by controlling the wettability of the seed surface by the expelled monomer. Very small monodisperse cross-linked polymer particles are initially prepared by RAFT mediated emulsion polymerization, then swollen with monomer and further polymerized to form anisotropic particles with a long dimension as little as $25 \mathrm{~nm}$. Both the shape of the anisotropic bulge and the polymer composition, of each end of the final Janus nanoparticle can be finely controlled. The surface active properties of the Janus nanoparticles are demonstrated by their ability to contribute as stabilizers and influence particle formation in a surfactant free ab-initio emulsion polymerization. The method provides a simple and reproducible process for the production of Janus colloidal nanoparticles readily achievable in a normal latex plant where batch size would be limited only by the size of the reactor.

Received 15th August 2014 Accepted 23rd September 2014 DOI: $10.1039 / c 4 p y 01125 b$ www.rsc.org/polymers is raised and expel some of the solvent. When the solvent is monomer, that monomer can be subsequently polymerized to form an anisotropic bulge on the surface of the original crosslinked particle. Our group has previously developed a method to form anisotropic polystyrene particles on a submicron scale (of order 300 to $500 \mathrm{~nm}$ in diameter). ${ }^{23}$ Although the particles formed by this process were anisotropic in shapes, they were not anisotropic particles in other respects as the particle surface and chemical composition was relatively uniform throughout each particle. In addition to the complicated and multiple step synthesis of Janus particles, typically at a very low solid content, the micron and sub-micron size of the Janus particles imposes significant limitations in the application of the Janus particles in bio-medical fields. ${ }^{24}$

Reversible Addition Fragmentation chain Transfer (RAFT) is a versatile controlled radical process that is applicable to a wide range of monomers at very accessible polymerization temperatures. ${ }^{25}$ Our group has pioneered the use of RAFT to mediate the emulsion polymerization processes. $^{26-31}$ Formation of aqueous based particles by living radical polymerization, especially by RAFT polymerization, has proved to be a very versatile and powerful technique to produce a range of particle morphologies and coating nanotechnology. ${ }^{32-34}$ The particle formation mechanism for the RAFT mediated

\footnotetext{
${ }^{a}$ Key Centre for Polymers and Colloids, School of Chemistry - F11, The University of Sydney, NSW 2006, Australia.E-mail: brian.hawkett@sydney.edu.au;

Fax: +61 29351 8651; Tel: +61 293516973

${ }^{b}$ DuluxGroup, 1970 Princess Hwy, Clayton VIC 3168, Australia
} 
emulsion polymerization process has recently been investigated and the knowledge gained enables us to exert very fine control over the desired properties of the particles formed. ${ }^{35}$ This approach is based on forming micelles from amphiphilic macro-RAFT agents, which are all turned into monodisperse latex particles that can be less than $20 \mathrm{~nm}$ in diameter.

In this study, we report a facile and robust water-based synthesis of polymeric anisotropic and Janus nanoparticles (NPs) that are appreciably smaller than previously reported. The particles can have longitudes of as short as $25 \mathrm{~nm}$, which can consist of two halves of different surface properties and/or different polymer compositions, using RAFT mediated emulsion polymerization. We also demonstrate the surface active properties of these Janus nanoparticles by controlling the size of latex particles in a surfactant free $a b$-initio emulsion polymerization.

\section{Experimental section}

\section{Materials}

Milli-Q water was used in the synthesis of all latexes. Acrylic acid (AA, Sumika) and 1,4-dioxane (Fluka) were distilled under reduced pressure. Styrene (Sty, Synthetic Resins), divinyl benzene (DVB, Fluka), butyl acrylate (BA, Aldrich) and methyl methacrylate (MMA, Aldrich) were passed through an inhibitor removal column (Aldrich). 2,2'-Azobis-isobutyronitrile (AIBN, Fluka) was recrystallized from ethanol. Acrylamide (AAm, Aldrich), 4,4'-azobis(4-cyanovaleric acid) (V-501, Wako), potassium persulfate (KPS, Aldrich), sodium dodecyl sulphate (SDS, Aldrich), sodium hydroxide (NaOH, Aldrich), methanol (MeOH, Ajax, HPLC grade) and tetrahydrofuran (THF, Ajax, HPLC grade) were used as received. The RAFT agent $2-\{[$ (butylsulfanyl)carbonothioyl]-sulfanyl\}propanoic acid (PABTC, Scheme 1A), was synthesized as previously described. ${ }^{26}$

\section{Analysis}

Electrospray mass spectrometry was carried out on a Finnigan LCQ MS detector with Finnigan LCQ Data Processing using Instrument Control Software. $10 \mathrm{uL}$ of solution $\left(10 \mu \mathrm{g} \mathrm{mL} \mathrm{m}^{-1}\right.$ in methanol or methanol-water) was injected.

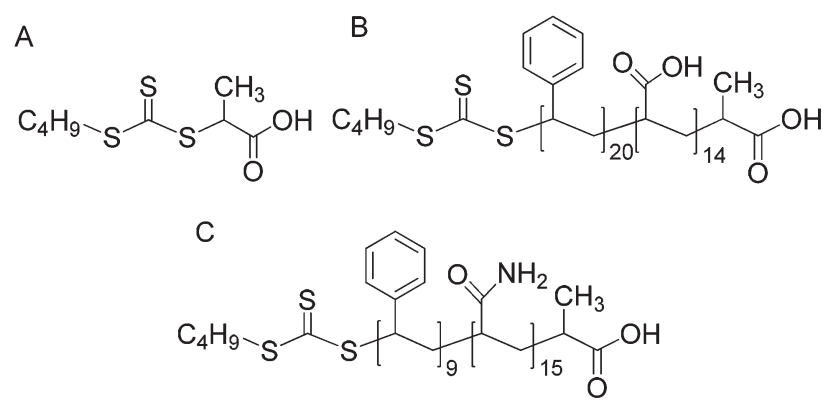

Scheme 1 Structure of RAFT agent (A) and RAFT diblock of styrene and acrylic acid (B) or styrene and acrylamide (C).
Dynamic light scattering (DLS): a Zetasizer NS, Malvern Instruments with a detection angle of $173^{\circ}$ was used to measure particle size (hydrodynamic diameter Z-Ave) and polydispersity index (PDI).

Hydrodynamic chromatography (HDC): a Particle Size Distribution Analyzer (Polymer Laboratories) with a PSDA-Type 2 column, PL-PSDA eluent at a flow rate of $1.7 \mathrm{~mL} \mathrm{~min}^{-1}$ was used to measure particle size distribution. Polystyrene latexes standards (Duke Scientific Corporation, from 20 to $350 \mathrm{~nm}$ diameter) were used for the calibration.

Transmission electron microscopy (TEM) images were recorded on the Philips CM120 Biofilter, Electron Microscope Unit, University of Sydney, using a CCD camera and an accelerating voltage of $120 \mathrm{kV}$. The samples ( $c a .0 .01 \mathrm{wt} \%$ in water) were deposited on carbon coated copper grids and dried under ambient conditions. $\mathrm{RuO}_{4}$ was used as a positive stain for polystyrene.

Gel permeation chromatography (GPC, Shimadzu) was used to measure molecular weights and molecular weight distributions of RAFT-Sty ${ }_{20}-b$ - $\mathrm{AA}_{14}$ diblocks. The method has been described previously. ${ }^{26,28,29}$

\section{Synthesis of amphiphilic macro-RAFT agents}

Synthesis of amphiphilic macro-RAFT diblock: RAFT-Sty $\mathbf{2 0}_{\mathbf{2}} \boldsymbol{b}$ $\mathbf{A A}_{14}$. Acrylic acid and styrene were polymerized in the presence of PABTC, in dioxane, to yield an amphiphilic diblock copolymer (Scheme 1B). The characterization of this diblock has been described elsewhere. ${ }^{28,29}$

Stage one, synthesis of the hydrophilic acrylic acid block: RAFT agent $\left(0.80 \mathrm{~g}, 3.36 \times 10^{-3} \mathrm{~mol}\right)$, V501 (0.05 g, $0.17 \times 10^{-3}$ mol), acrylic acid (4.84 g, $\left.67.15 \times 10^{-3} \mathrm{~mol}\right)$ and dioxane $(22.03 \mathrm{~g})$ were mixed in a round-bottom flask. The mixture was stirred at room temperature until both RAFT agent and initiator were completely dissolved, sparged with nitrogen for 20 minutes and then immersed in an $80^{\circ} \mathrm{C}$ oil bath under constant stirring for 2 hours. The flask was then cooled down to room temperature and a sample was withdrawn for electrospray mass spectrometry.

Stage two, synthesis of the hydrophobic block: styrene (10.25 g, $\left.98.45 \times 10^{-3} \mathrm{~mol}\right), \mathrm{V}-501\left(0.24 \mathrm{~g}, 0.84 \times 10^{-3} \mathrm{~mol}\right)$ and $24.52 \mathrm{~g}$ dioxane were added. The mixture was again sparged with nitrogen and magnetically stirred in an $80^{\circ} \mathrm{C}$ oil bath under constant stirring for at least 2 hours. The length of styrene hydrophobic block was checked by gravimetry and GPC. Most of the dioxane was removed from the final copolymer solutions by sparging nitrogen through the solution while it was being stirred overnight on a magnetic stirrer in a fume hood. The copolymer was then dried in a vacuum oven at $60{ }^{\circ} \mathrm{C}$ until solvent-free copolymer was obtained. Yellow powder of RAFT-Sty ${ }_{20} \mathrm{AA}_{14}$ amphiphilic diblock was obtained.

Synthesis of amphiphilic macro-RAFT diblock: RAFT-Sty $\mathbf{9}_{\mathbf{9}} \boldsymbol{b}$ $\mathbf{A A m}_{\mathbf{1 5}}$. Acrylamide and styrene were polymerized in the presence of PABTC, in a dioxane-water mixture, to yield an amphiphilic diblock copolymer (Scheme 1C).

Stage one, synthesis of the hydrophilic block: RAFT agent $\left(0.80 \mathrm{~g}, 3.36 \times 10^{-3} \mathrm{~mol}\right), \mathrm{V}-501\left(0.10 \mathrm{~g}, 0.36 \times 10^{-3} \mathrm{~mol}\right)$, 
acrylamide $\left(3.71 \mathrm{~g}, 52.21 \times 10^{-3} \mathrm{~mol}\right)$, water $(4.41 \mathrm{~g})$ and dioxane $(6.61 \mathrm{~g})$ were mixed in a round-bottom flask. The mixture was stirred at room temperature (until both RAFT agent and initiator were completely dissolved), sparged with nitrogen for 20 minutes, immersed in a $70{ }^{\circ} \mathrm{C}$ oil bath and magnetically stirred for 5 hours. The flask was then cooled down to room temperature. The length of the acrylamide block was determined by electrospray mass spectrometry.

Stage two, synthesis of the hydrophobic block: styrene (3.16 g, $\left.30.32 \times 10^{-3} \mathrm{~mol}\right)$, V501 (0.19 g, $\left.0.69 \times 10^{-3} \mathrm{~mol}\right)$, water $(6.14 \mathrm{~g})$ and dioxane $(21.15 \mathrm{~g})$ were added to the macro-RAFT solution $(0.80 \mathrm{~g})$ from stage 1 to obtain a clear solution. The mixture was sparged with nitrogen for 10 minutes, immersed in a $70{ }^{\circ} \mathrm{C}$ oil bath and magnetically stirred overnight. Conversion was checked by gravimetry.

\section{Formation of Janus nanoparticles (Janus NPs) from cross- linked polystyrene seed particles stabilized by RAFT-Sty Ro $^{-} b$ - $\mathbf{A A}_{14}$}

Two different types of cross-linked polystyrene (PSty) seed nanoparticles stabilized by the poly(acrylic acid)- $b$-polystyrene macro-RAFT agents were synthesized, one using V-501 as initiator and the other KPS. The particles were expected to have a more hydrophilic surface when KPS was used as initiator relative to when V501 was used.

\section{Synthesis of DVB cross-linked PSty seeds using V-501 as initiator (V-501 derived PSty seeds)}

Stage one, formation of micelles (Scheme 2, step 1): a mixture of RAFT-Sty ${ }_{20} \mathrm{AA}_{14}$ amphiphilic diblock $(0.50 \mathrm{~g})$, sodium hydroxide $(0.15 \mathrm{~g})$ and water $(15 \mathrm{~g})$ was stirred on a magnetic stirrer for 60 minutes, followed by sonication in a sonic bath until a clear yellow micellar solution was obtained, with the diameter Z-Ave of $19.9 \mathrm{~nm}$ by DLS or the average volume mean diameter of $21 \mathrm{~nm}$ with the coefficient of variance of $7.8 \%$ by HDC (see Table 1).

Stage two, extension of hydrophobic block length to lock in micelles to form particles (Scheme 2, step 2): To the micellar solution $(2.46 \mathrm{~g})$ in a $25 \mathrm{ml}$ round bottom flask, styrene $(0.25 \mathrm{~g})$ and water $(8.03 \mathrm{~g})$ were then added and stirred overnight. Initiator $\mathrm{V}-501(0.01 \mathrm{~g})$ was added to the monomer swollen micelles. The flask was sealed, sparged with nitrogen for 15 minutes and immersed in an oil bath with a temperature setting of $80^{\circ} \mathrm{C}$ for 4 hours under constant magnetic stirring. The reaction reached approximately $80 \%$ conversion.

Stage three, formation of crosslinked particles (Scheme 2, step 3), (Since the particles are largely made up of living polymers we are able to conduct the crosslinking step after the particles have been formed): DVB $(0.10 \mathrm{~g})$ and initiator AIBN $(0.014 \mathrm{~g})$ were then added. The flask was stirred to mix for 2 hours at room temperature and immersed overnight in an oil bath with a temperature setting of $75{ }^{\circ} \mathrm{C}$ under constant magnetic stirring. The final latex particles had an average diameter of $27.6 \mathrm{~nm}$ by DLS and the volume mean diameter of $25.8 \mathrm{~nm}$ (coefficient of variance of $7.9 \%$ ) by HDC. Transmission electron microscopy showed that the latex contained nanoparticles with narrow size distribution with a diameter of approximately $17 \mathrm{~nm}$ (Fig. 1A).

\section{Synthesis of DVB cross-linked PSty seeds stabilized by polyAA chains and initiator derived sulfate groups (KPS derived PSty seeds)}

Stage one, formation of micelles (Scheme 2, step 1): a mixture of RAFT-Sty ${ }_{20} \mathrm{AA}_{14}$ amphiphilic diblock (0.379 g), sodium hydroxide $(0.075 \mathrm{~g})$ and water $(7.57 \mathrm{~g})$ in a $25 \mathrm{~mL}$ round bottom flask was stirred on a magnetic stirrer for 60 minutes, followed by sonication in a sonic bath for 30 minutes.

Stage two, extension of hydrophobic block length to lock in micelles to form particles (Scheme 2, step 2): To the micellar
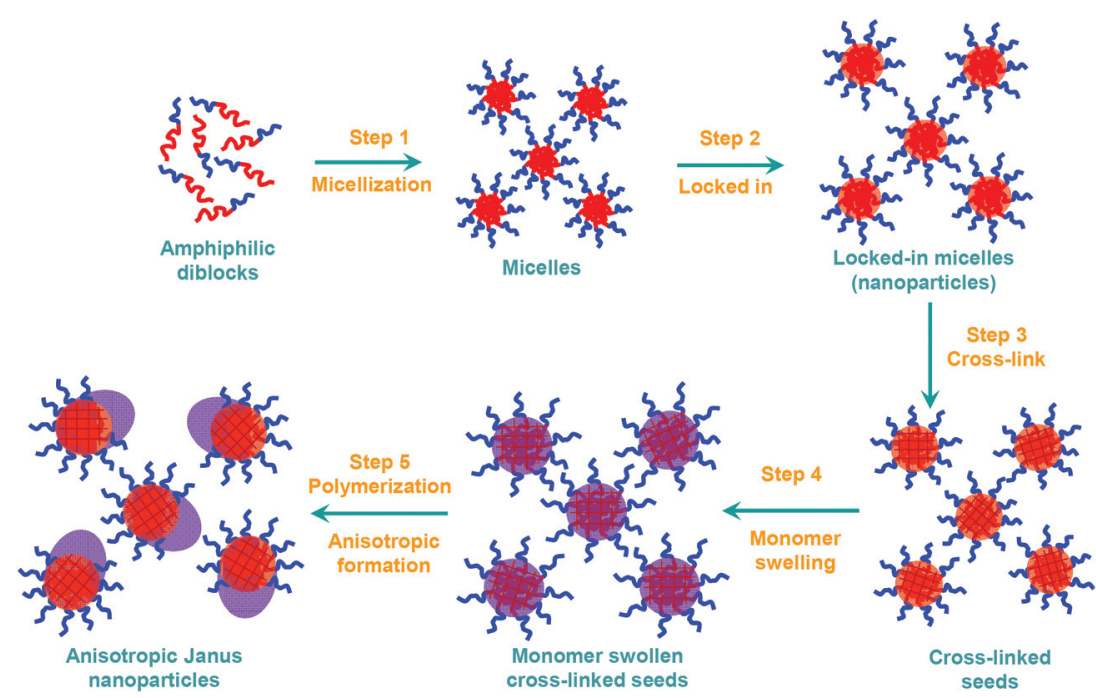

Scheme 2 Schematic of the procedure for the synthesis of anisotropic and Janus nanoparticles: the morphology of the final particles depends on the properties of cross-linked seeds, on the initiator and monomer(s) used in the polymerization. 
Table 1 Particle size and distributions of micelles from RAFT-Sty ${ }_{20} \mathrm{AA}_{14}$ diblocks and the corresponding seeds from the DLS measurements (Zetasizer, Malvern Instrument) and from the HDC (Polymer Laboratories)

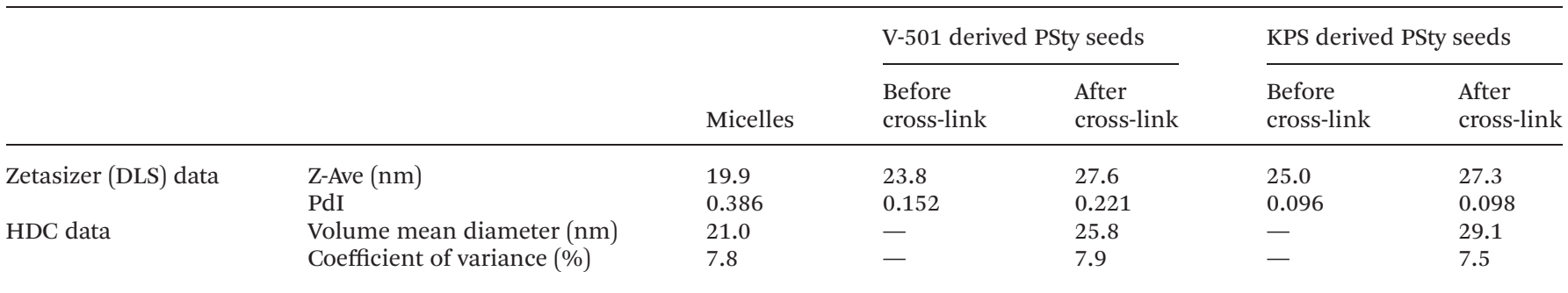
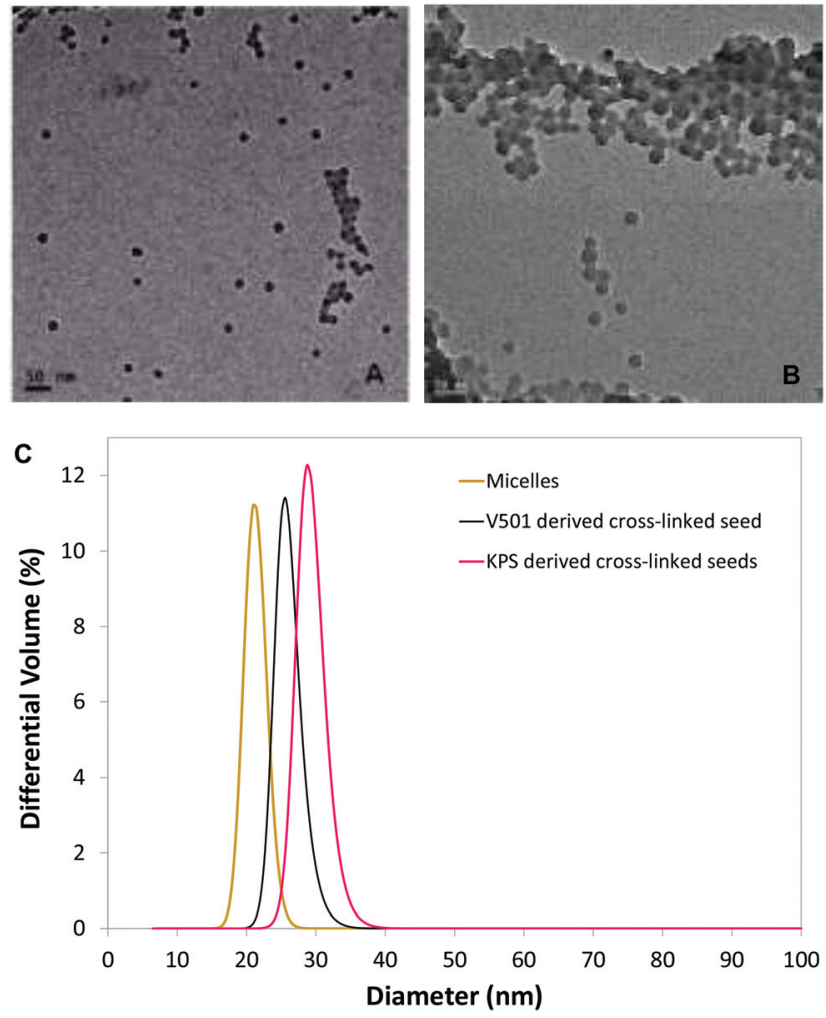

Fig. 1 Characterisations of the cross-linked PSty seeds which were synthesized from RAFT-Sty $20 \mathrm{AA}_{14}$ diblocks via micellization: TEM micrographs of the cross-linked V-501 derived PSty seeds (A) and the crosslinked KPS derived PSty seeds (B); (C) particle size distributions by HDC measurements for the starting $\operatorname{Sty}_{20} \mathrm{AA}_{14}$ micelles and the cross-linked seeds $A$ and $B$. The coefficient of variance of less than $8 \%$ in all measurements indicated that micelles and their corresponding seeds had narrow size distributions.

solution, styrene $(0.855 \mathrm{~g})$ and sodium hydrogen carbonate $(8.7 \mathrm{mg})$ were added and stirred overnight. Initiator KPS $(0.02 \mathrm{~g})$ was added to the monomer swollen micelles. The flask was sealed, sparged with nitrogen for 15 minutes and immersed in an oil bath with a temperature setting of $80^{\circ} \mathrm{C}$ for 4 hours under constant magnetic stirring. The reaction reached approximately $80 \%$ conversion.

Stage three, formation of crosslinked particles (Scheme 2, step 3): DVB (0.346 g) and initiator AIBN (0.032 g) were then added, stirring to mix for 1 hour at room temperature. The flask was then immersed overnight in an oil bath with a temperature setting of $75^{\circ} \mathrm{C}$ under constant magnetic stirring. The final latex particles had an average diameter of $27.3 \mathrm{~nm}$ by DLS and the volume mean diameter of $29.1 \mathrm{~nm}$ (coefficient of variance of $7.5 \%$ ) by HDC. Transmission electron microscopy showed that the latex contained nanoparticles with narrow size distribution (Fig. 1B).

\section{Synthesis of ellipsoidal nanoparticles using the V-501 derived seed latex (Scheme 2, step 4)}

A mixture of the $\mathrm{V}-501$ derived polystyrene seed latex $(6.02 \mathrm{~g})$, milliQ water (9.75 g), styrene monomer (1.62 g) and SDS $(0.11 \mathrm{~g})$ was prepared in a $25 \mathrm{~mL}$ round bottom flask and stirred overnight. Initiator V-501 (0.068 g) was added to the monomer swollen latex particles. The flask was sealed, stirred at room temperature for 30 minutes and sparged with nitrogen for 15 minutes and immersed in an oil bath with a temperature setting of $80{ }^{\circ} \mathrm{C}$ under constant magnetic stirring for 4 hours. TEM micrographs as shown in Fig. 2A showed that the final latex contained monodisperse ellipsoidal nanoparticles with average dimensions of $23 \mathrm{~nm}$ width and $35 \mathrm{~nm}$ length.

\section{Synthesis of Janus nanoparticles using the KPS derived seed latex (Scheme 2, step 4)}

Synthesis of snowmen shaped nanoparticles with polystyrene bulge. A mixture of the KPS derived polystyrene seed latex $(2.25 \mathrm{~g})$, styrene monomer $(0.52 \mathrm{~g})$ and water $(5.17 \mathrm{~g})$ were prepared in a $25 \mathrm{~mL}$ round bottom flask and stirred overnight. Initiator AIBN (0.015 g) was added to the monomer swollen latex particles. The flask was sealed, stirred at room temperature for 2 hours and sparged with nitrogen for 10 minutes. The flask was then immersed in an oil bath with a temperature setting of $75^{\circ} \mathrm{C}$ under constant magnetic stirring for 5 hours. TEM micrographs showed that the final latex contained snowmen shaped nanoparticles having the longitude of less than $100 \mathrm{~nm}$ and narrow particle size distribution (see Fig. 2B and $\mathrm{C}$ ).

Synthesis of dumbbell shaped nanoparticles with poly(acrylate/methacrylate) bulge. A mixture of the KPS derived polystyrene seed latex (1.95 g), methyl methacrylate and butyl acrylate monomer mixture at the weight ratio of $7: 3(0.50 \mathrm{~g})$ and water $(4.68 \mathrm{~g})$ were prepared in a $25 \mathrm{~mL}$ flask and stirred overnight. Initiator AIBN (0.015 g) was added to the monomer swollen latex particles and the flask was sealed, stirred at room 

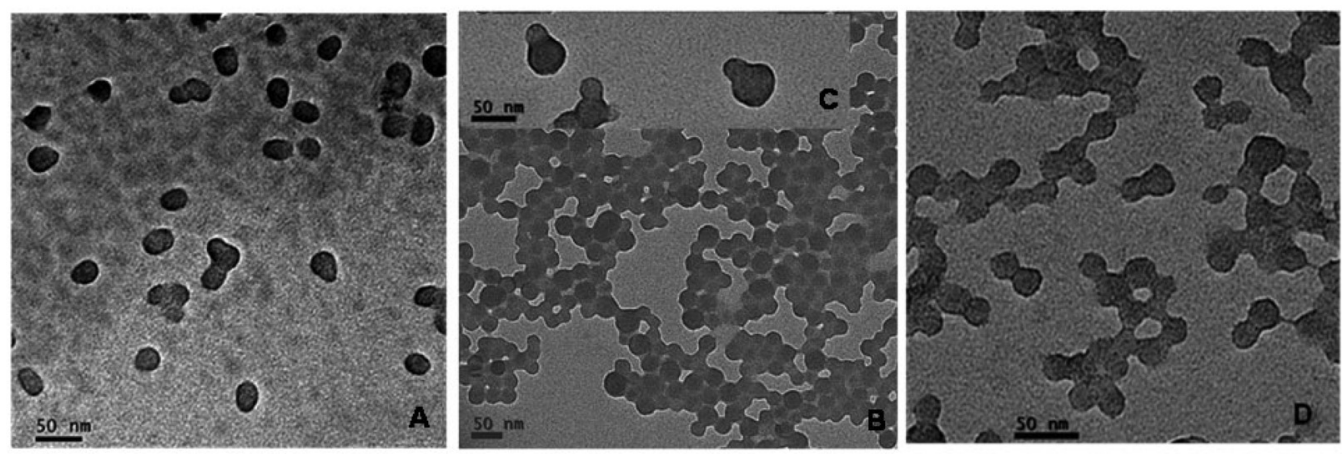

Fig. 2 TEM micrographs of (A) ellipsoidal Janus nanoparticles with both halves of PSty, which were synthesized from the cross-linked V-501 derived seeds (as shown in Fig. 1A); of (B, C) snowman shaped Janus nanoparticles with both ends of PSty or (D) dumbbell shaped particles with one half of polystyrene and the other of poly(butyl acrylate-co-methyl methacrylate), which were synthesized from the cross-linked KPS derived PSty seeds (as shown in Fig. 1B).

temperature for 2 hours, sparged with nitrogen for 10 minutes and immersed in an oil bath with a temperature setting of $75{ }^{\circ} \mathrm{C}$ under constant magnetic stirring for 5 hours. TEM micrographs showed that the final latex contained monodisperse dumbbell shaped nanoparticles with each half having an average diameter of approximately $23 \mathrm{~nm}$ (see Fig. 2D).

\section{Formation of Janus nanoparticles (Janus NPs) from a cross- linked AIBN derived PSty seed sterically stabilized by polyacrylamide (sterically stabilized seed latex)}

Synthesis of DVB cross-linked polystyrene nanoparticles sterically stabilized by polyAAm chains. Stage one and two, formation of monomer swollen micelles and extension of hydrophobic block length to form particles (Scheme 2, steps 1 and 2): The RAFT-Sty ${ }_{9} \mathrm{AAm}_{15}$ diblocks are very difficult to solubilize in water, their micelles were therefore prepared in the presence of styrene monomer. A mixture of the RAFTSty ${ }_{9} \mathrm{AAm}_{15}$ amphiphilic diblocks $(0.50 \mathrm{~g})$, water $(20 \mathrm{~g})$ and styrene monomer $(1.20 \mathrm{~g})$ in a $25 \mathrm{ml}$ round bottom flask was stirred on a magnetic stirrer overnight to obtain well dispersed yellowish emulsion. To the emulsion initiator AIBN (0.02 g) was added. The flask was sealed, sparged with nitrogen for 15 minutes and immersed in an oil bath with a temperature setting of $70^{\circ} \mathrm{C}$ under constant magnetic stirring for 5 hours.

Stage three, formation of crosslinked particles (Scheme 2, step 3): to a $50 \mathrm{~mL}$ round bottom flask containing the sterically stabilized polystyrene latex particles $(10.03 \mathrm{~g})$ DVB $(0.22 \mathrm{~g})$, initiator AIBN $(0.032 \mathrm{~g})$ and water $(2.42 \mathrm{~g})$ were then added and stirred to mix for 5 hours at room temperature. The flask was then sealed, sparged with nitrogen for 15 minutes and immersed in an oil bath with a temperature setting of $75{ }^{\circ} \mathrm{C}$ under constant magnetic stirring overnight. The final latex particles had an average diameter of $109 \mathrm{~nm}$ and PDI of 0.078 by light scattering. Transmission electron microscopy showed that the latex contained polyacrylamide stabilized nanoparticles with low polydispersity (Fig. 3A).

Synthesis of ellipsoidal nanoparticles using the sterically stabilized seed latex (Scheme 2, step 4). A mixture of the latex formed in step 3, containing cross-linked sterically stabilized

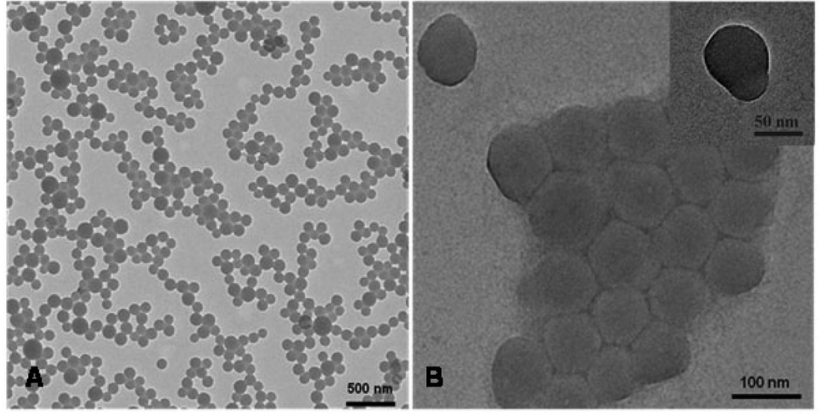

Fig. 3 TEM micrographs of (A) cross-linked polystyrene seed nanoparticles sterically stabilized by polyacrylamide and (B) anisotropic Janus nanoparticles with both halves of polystyrene, which were synthesized from the cross-linked seeds in (A).

polystyrene nanoparticles (12.71 g), water (6.76 g), styrene monomer $(0.42 \mathrm{~g})$ and sodium dodecyl sulphate SDS (0.10 g) was prepared in a $25 \mathrm{~mL}$ round bottom flask and stirred at room temperature for 3 hours. Initiator V-501 (0.07 g) was added to the monomer swollen latex particles and the flask sealed, sparged with nitrogen for 15 minutes and immersed in an oil bath with a temperature setting of $75^{\circ} \mathrm{C}$ under constant magnetic stirring for 3 hours. Light scattering showed that the final anisotropic particles had an average diameter of $116 \mathrm{~nm}$ and PDI of 0.081. TEM micrographs (see Fig. 3B) showed that the final polystyrene particles had ellipsoidal morphology in which one half was sterically stabilized by polyacrylamide macro-RAFT.

Synthesis of surfactant free polystyrene or poly(butyl acrylateco-methyl methacrylate) particles using the Janus nanoparticles as stabilizers affecting particle sizes of the final latex particles

Recipes used to synthesize polystyrene or poly(butyl acrylateco-methyl methacrylate) latex particles in a surfactant-free ab-initio emulsion polymerization are given in Table 2 . As a proof-of-concept experiment, only one type of Janus polystyrene nanoparticles, which were formed from the cross-liked 
Table 2 Ab-initio emulsion polymerizations of styrene or (butyl acrylate-co-methyl methacrylate) using Janus polystyrene nanoparticles which have $\mathrm{V}$-501 derived cross-linked polystyrene seeds stabilized by RAFT-Sty ${ }_{20} \mathrm{AA}_{14}$

\begin{tabular}{|c|c|c|c|c|c|c|}
\hline Particles & $\begin{array}{l}\text { Janus particles } \\
\text { (wt } \% \text { to monomer) }\end{array}$ & $\begin{array}{l}\text { Monomer } \\
\text { mass }(\mathrm{g})\end{array}$ & $\begin{array}{l}\text { V-501 } \\
(\mathrm{mM})\end{array}$ & $\begin{array}{l}\mathrm{NaOH} \\
(\mathrm{mM})\end{array}$ & $\begin{array}{l}\mathrm{H}_{2} \mathrm{O} \\
(\mathrm{g})\end{array}$ & $\begin{array}{l}\text { Conversion by } \\
\text { gravimetry (\%) }\end{array}$ \\
\hline PSty-1 & 0 & 5.6035 & 15.0 & 106 & 22.29 & 75 \\
\hline $\mathrm{P}(\mathrm{BA}-c o-\mathrm{MMA})-1$ & 0 & 1.4276 & 16.1 & 125 & 10.41 & 70 \\
\hline $\mathrm{P}(\mathrm{BA}-c o-\mathrm{MMA})-2$ & 1 & 2.8408 & 13.0 & 127 & 23.41 & 87 \\
\hline $\mathrm{P}(\mathrm{BA}-c o-\mathrm{MMA})-3$ & 5 & 1.1252 & 12.6 & 120 & 11.51 & 85 \\
\hline
\end{tabular}

V-501 derived polystyrene seeds using RAFT-Sty ${ }_{20} \mathrm{AA}_{14}$ diblocks, was employed.

A desired amount of Janus particle dispersion, styrene or a mixture of (butyl acrylate-co-methyl methacrylate) (1/1 mass ratio), $\mathrm{NaOH}$, water and $\mathrm{V}-501$ as initiator were weighed into a $50 \mathrm{~mL}$ round bottom flask. The flask was sealed, sparged with nitrogen for 15 minutes and immersed in an oil bath with a temperature setting of $70{ }^{\circ} \mathrm{C}$ under constant magnetic stirring overnight. The final conversion was determined by gravimetry. The particle size and morphology of the final latex particles was determined by DLS (or HDC) and TEM, respectively.

\section{Discussions}

The formation of polymeric nanoparticles from micelles in ab initio RAFT mediated emulsion polymerization has been previously studied in our group. ${ }^{35}$ In this study, micelles were first formed from the amphiphilic macro-RAFT-Sty ${ }_{20} \mathrm{AA}_{14}$ diblocks. These micelles were then further polymerized using either V-501 or KPS as an initiator to form poly(acrylic acid) stabilized PSty seeds, using recipes stated in the Experimental section. Our previous studies showed that polymerization using these diblocks under similar conditions were under RAFT control. ${ }^{28,29}$ The particle size and size distribution of the micelles and PSty seeds by DLS and HDC is presented in Table 1. The HDC particle sizing method is based on the principle of chromatography that the smaller particles are eluted after the bigger ones. The elution is independent of the particle morphology. The results given by the HDC measurements for the micelles and cross-linked seeds (see Fig. 1C) showed that they all had narrow size distribution with the coefficient of variance of less than $8 \%$. The particle size distribution was further investigated using the TEM. TEM micrographs (see Fig. 1A and B) showed that both types of cross-linked PSty seeds had narrow particle size distribution. The cross-linked V-501 derived seeds (see Fig. 1A) appeared slightly smaller in size than the cross-linked KPS derived seeds (see Fig. 1B), in a good agreement with the TEM result.

The cross-linked PSty seeds were then used to synthesize Janus nanoparticles. The particle morphologies obtained when polymerizing monomer swollen cross-linked seed particles, depends on the surface wetting of the seed particle by the polymerizing monomer, which has been discussed in detail by Mock et $a .^{23}$ Scheme 3 illustrates a number of

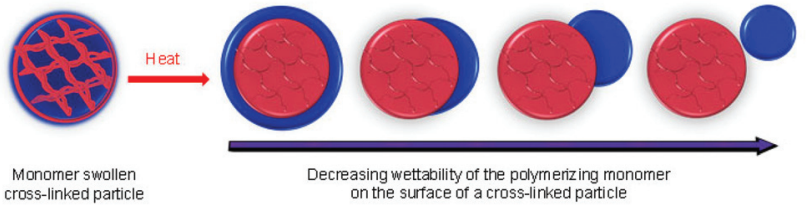

Scheme 3 Schematic behaviour of the monomer swollen cross-linked polymer seed on heating. The wetting ability of the monomer governs the morphology of the anisotropic particles formed during subsequent polymerization. In the reactions described here, complete detachment of a new particle is prevented by RAFT control making the new polymer continuous with the old.

possible morphologies that can be formed during the subsequent polymerization of the swelling monomer.

The wettability of the seed surface by the polymerizing monomer(s) can be controlled by the type of stabilizer used to prepare the cross-linked seed particles and the type of initiator used in their preparation. When AIBN was used as the initiator no charged initiator residues were expected on the surface and the particle surface between the stabilizers was expected to be most hydrophobic. Using V501 initiator to yield carboxylic acid residues on the surface was expected to produce a more hydrophilic surface, while the sulphate residues derived from the persulphate initiator KPS was expected to yield a more hydrophilic surface again. The stabilisers derived from the original micelles are expected to play a very limited role as they are very sparsely distributed over the surface by the time the micelles are grown up tp crosslinked seeds. The compatibility of the monomer used to swell the crosslinked seed particles with their surface was further changed by changing the monomer from styrene to a blend of MMA and BA. TEM was used to examine morphologies of the claimed Janus nanoparticles.

When Janus NPs were synthesised from the two crosslinked seed particles in accordance with Scheme 2, step 4, the polymerizing monomer from the monomer swollen particles was expelled and protruded out of the original seed particles, forming different bulge configurations based on the interactions between the expelled monomer and the surface of the seed particles. When V-501 was used as an initiator to grow the cross-linked seed particles (Scheme 2, step 3), the polymerization of the swollen styrene formed PSty Janus NPs with ellipsoidal shapes (see Fig. 2A). This indicated that, despite the carboxylic charge derived from the V501 on the surface, 
the expelled styrene monomer wetted the surface reasonably well.

When the cross-linked KPS derived seed particles were used to grow Janus NPs with both ends of PSty, snowman shaped anisotropic particles were obtained (see Fig. 2B, and the insert $2-\mathrm{C})$. This is consistent with our original hypothesis that these particles would be less easily wetted by the styrene monomer than those obtained from the V-501 derived seeds. When styrene was replaced by a blend of MMA/BA monomers to swell the cross-linked KPS derived seeds, we were initially surprised to observe the formation of dumbbell shaped Janus NPs (see Fig. 2D) on the polymerization. This blend was expected to be more hydrophilic than pure styrene but the particle morphology indicated poorer surface wetting. It seems that we were under estimating the influence of the compatibility of the underlying polymer surface with the polymer of the newly formed bulge. Indeed, the incompatibility and phase separation of the two polymers may well have forced a complete detachment of the newly formed bulge had it not have been for many of the acrylic/methacrylic polymer chains still being linked to the original seed particle because the whole particle was grown under some measure of RAFT control.

Moving from the electrostatic stabilized to sterically stabilized seeds, RAFT-Sty ${ }_{9} \mathrm{AAm}_{15}$ diblocks were used instead of the -RAFT-Sty ${ }_{20} \mathrm{AA}_{14}$ diblocks. However, the RAFT-Sty ${ }_{9} \mathrm{AAm}_{15}$ amphiphilic diblock is not dispersible in the aqueous phase due to the hydrophobicity imparted by the styrene block. We therefore employed a small amount of styrene monomer to solubilize the styrene block and enabled the formation of the monomer swollen micelles from the RAFT-Sty ${ }_{9} \mathrm{AAm}_{15}$ amphiphilic diblock. These monomer swollen micelles were polymerized and formed sterically stabilized PSty seeds with Z-Ave diameter of $84 \mathrm{~nm}$, PDI 0.16. The TEM micrograph shows that we achieved a reasonably narrow particle size distribution for the polyacrylamide (sterically) stabilized PSty seeds (see Fig. 3A).

As the polyacrylamide stabilized crosslinked seeds were prepared using AIBN as an initiator there would have been no initiator derived charge on the surface. Only the polyacrylamide chains would have reduced the compatibility of the surface with the expelled monomer. The ellipsoidal shape of the derived Janus NPs suggests that the incompatibility of the polyacrylamide with both the styrene monomer and the polymer formed from it was such as to prevent the complete wetting of the entire seed surface and enable the formation of ellipsoidal Janus NPs.

Another important achievement from all processes was that no secondary population of new particles was observed in any experiments. Particle size and particle size distribution measurements by HDC (see Fig. 5) showed that the monodisperse crosslinked PSty seeds led to monodisperse ellipsoidal Janus NPs.

The synthesized Janus NPs are expected to impose surface active properties due to the anisotropic surface properties of the two halves. Based on the principles of emulsification and emulsion polymerization, surface active molecules influence particle/droplet sizes of emulsion droplets and/or of polymerizing particles.
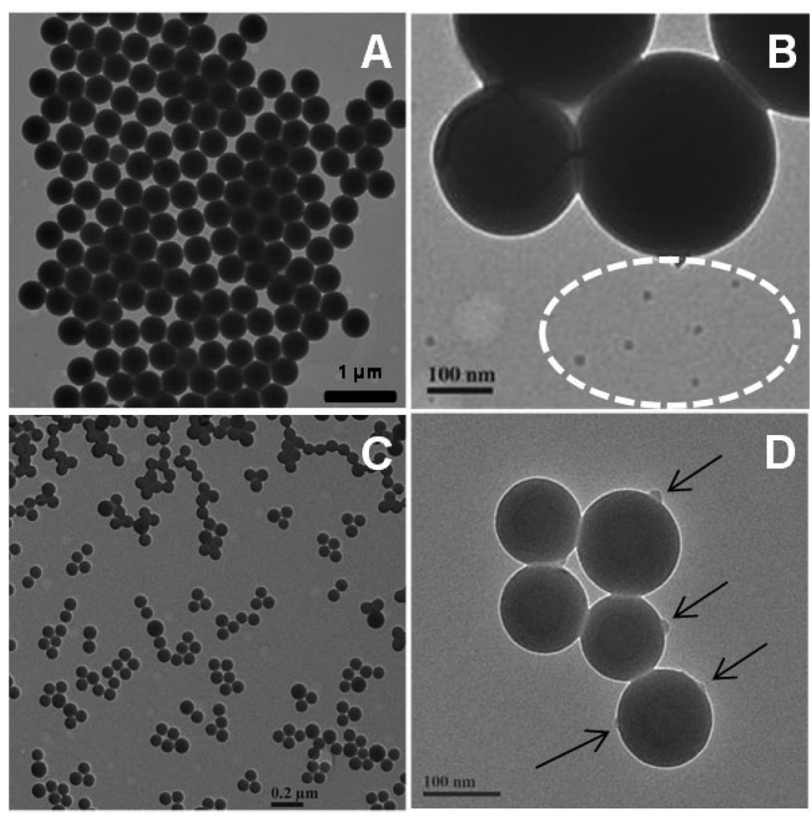

Fig. 4 TEM micrographs of the surfactant free PSty-1 particles in the absence ( $A$ and $B$ ) and PSty-2 particles in the presence ( $C$ and $D$ ) of the Janus NPs. Janus NPs were obtained from the cross-linked V-501 derived PSty seeds shown in Fig. 2A.

For a proof of concept characterisation, we used the synthesised Janus NPs as stabilising agents in a surfactant free emulsion polymerization of styrene or acrylate/methacrylate monomers to study the influence of Janus NPs on particle formation and final particle size. The ellipsoidal Janus NPs (as shown in Fig. 2A) with one half stabilised by poly(acrylic acid) were employed in all surfactant-free syntheses (see Table 2). If the Janus NPs were surface active, the Janus NPs were expected to localize on the surface of a latex particle during surfactant free emulsion polymerization, with poly(acrylic acid) stabilised half protruding further out into to the aqueous phase.

Using the recipe given in Table 2, the surfactant free PSty particles (PSty-1) had an average diameter of $610 \mathrm{~nm}$ by light scattering. Our previous study found out that the PSty particles in a surfactant free ab-initio emulsion polymerization were formed by the aggregation of smaller precursor particles. ${ }^{36}$ In this case a population of the small precursor particles that were not collected up into the main population of larger particles were also observed in the TEM micrograph (see Fig. 4A and $\mathrm{B}$, indicated by dotted circle). In the presence of Janus NPs at a concentrations as low as $1 \mathrm{wt} \%$ (based on monomer mass), the final PSty particles (PSty-2) were significantly reduced in size and had a narrower size distribution (see Table 3). The TEM micrograph (Fig. 4C and D) shows the presence of Janus NPs on the surface of PSty- 2 particles (black arrows) with a maximum of 2 Janus NPs on each PSty-2 particle. In some PSty-2 particles, Janus NPs were not apparent, probably due to their hidden position on the TEM grid when dried.

In TEM studies, poly(acrylates/methacrylates) have much lower electron density than polystyrene especially when the 
Table 3 DLS (Zetasizer, Malvern Instrument) and HDC (Polymer Laboratories) measurements for particle size and distributions of the $a b$-initio surfactant free latex particles with and without a presence of Janus NPs. (PDI: polydispersity index; CV: coefficient of variance)

\begin{tabular}{llll}
\hline & $\begin{array}{l}\text { wt\% of } \\
\text { Janus NPs } \\
\text { (to monomer) }\end{array}$ & $\begin{array}{l}\text { Z-Ave diameter } \\
\text { by Zetasizer } \\
(\mathrm{nm})\end{array}$ & $\begin{array}{l}\text { Volume mean } \\
\text { diameter by } \\
\text { HDC }(\mathrm{nm})\end{array}$ \\
\hline PSty-1 & 0 & $610(\mathrm{PDI}=0.207)$ & - \\
PSty-2 & 1 & $215(\mathrm{PDI}=0.045)$ & $196.2(\mathrm{CV}=17.5 \%)$ \\
P(BA-co-MMA)-1 & 0 & $812(\mathrm{PDI}=0.374)$ & - \\
P(BA-co-MMA)-2 & 1 & $229(\mathrm{PDI}=0.027)$ & $186.7(\mathrm{CV}=12.4 \%)$ \\
P(BA-co-MMA)-3 & 5 & $147(\mathrm{PDI}=0.008)$ & $117.4(\mathrm{CV}=8.4 \%)$
\end{tabular}

PSty is positively stained (with $\mathrm{RuO}_{4}$ for example), which makes poly(BA/MMA) particles appear brighter than PSty particles. Styrene monomer was therefore replaced by a mixture of (BA/MMA) monomers to achieve a better contrast between the Janus NPs and the final latex particles. A mixture of $1 / 1$ mass ratio BA/MMA was used to obtain glassy copolymer particles which avoided coalescence on the TEM grid. The $\mathrm{P}\left(\mathrm{BA}-\mathrm{co}^{-}\right.$ MMA)-1 latex made by surfactant free emulsion polymerization without Janus NPs had a Z-average diameter of $812 \mathrm{~nm}$ and a broad particle distribution. Similar to PSty-2 particles, with $1 \mathrm{wt} \%$ of Janus NPs, P(BA-co-MMA)-2 particles were obtained with significantly smaller size and narrower size distribution than P(BA-co-MMA)-1 (see Table 3). P(BA-co-MMA)-2 and PSty-2 latex particles have similar size and size distribution. At a higher concentration of the Janus NPs, $5 \mathrm{wt} \%$ on monomer, $\mathrm{P}(\mathrm{BA}-\mathrm{co}-\mathrm{MMA})-3$ latex particles were further reduced in size and size distribution as shown in Table 3 and Fig. 5.

TEM micrographs (Fig. 6) of the P(BA-co-MMA) latex particles positively stained by $\mathrm{RuO}_{4}$ showed that when Janus NPs were present in the surfactant free emulsion polymerization, darker regions/dots appeared clearly on the surface of both $\mathrm{P}$ (BA-co-MMA)-2 and P(BA-co-MMA)-3 latex particles (Fig. 6B and C). These black dots were only given by the Janus polystyrene NPs. Similar to PSty-2, a maximum of 2 Janus NPs were

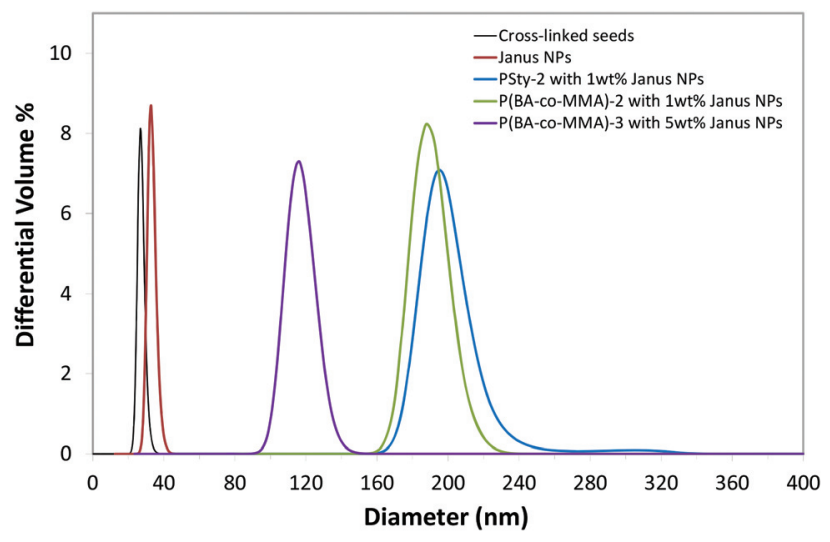

Fig. 5 HDC measurements for particle size distributions of the crosslinked seeds (shown in Fig. 1A), the corresponding Janus PSty NPs (shown in Fig. 2A) and the composite latex particles of PSty or P(BA-coMMA) from the surfactant free emulsion polymerizations in the presence of Janus NPs.

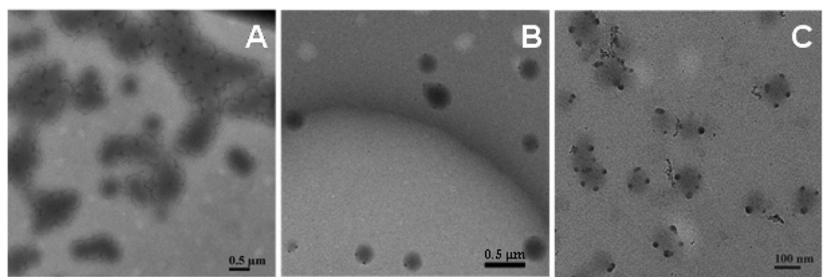

Fig. 6 TEM micrographs of the RuO4 stained surfactant free poly(BAco-MMA) particles: $\mathrm{P}(\mathrm{BA}-\mathrm{co}-\mathrm{MMA})-1$ without Janus NPs (A); $\mathrm{P}(\mathrm{BA}-\mathrm{CO}-$ MMA)-2 (B) and P(BA-CO-MMA)-3 (C) in the presence of 1 wt\% and 5 wt\% Janus NPs, respectively. Janus NPs were obtained from the cross-linked V-501 derived PSty seeds (shown in Fig. 2A).

observed on each $\mathrm{P}(\mathrm{BA}-c o-\mathrm{MMA})-2$ latex particle when using $1 \mathrm{wt} \%$ Janus NPs. When the concentration of the Janus NPs was increased to $5 \mathrm{wt} \%$, a maximum of 4 Janus NPs on each $\mathrm{P}$ (BA-co-MMA)-3 latex particle was found. The results presented here strongly support the surface active properties of our claimed Janus particles with two halves of different hydrophilic/hydrophobic surfaces. It was an unexpected and interesting discovery that it takes very few Janus nanoparticles to significantly influence the size of the particles that result from this surfactant free emulsion polymerization. The presence of this few Janus particles on the surface the final particle not only made the final composite particles much smaller but also more monodisperse. It is also interesting to note that there does not appear to be any Janus particles engulfed within the much larger surfactant free particles. The Janus NPs appear to play an important role in the nucleation and stabilization of the particles formed in an ab-initio surfactant free emulsion polymerization and have considerable control over particle size and particle size distribution. Further investigations are required to fully understand this exciting discovery.

\section{Conclusions}

We have demonstrated that monodisperse surfactant-free anisotropic Janus nanoparticles with a long dimension of as small as $25 \mathrm{~nm}$ with desired surface hydrophobicity/hydrophilicity and polymer compositions can be successfully designed, tuned and synthesized employing facile, reproducible and robust procedures, using RAFT mediated emulsion polymerizations. By controlling the wettability of the surface of the crosslinked seed particles by the swelling monomer(s) and/or the compatibility of the bulge polymer with the surface of the seed particles, we can control the morphology of the particle obtained on polymerization. The overall size and dimensions of the final Janus particles were controlled by adjusting the size of the original seed particles, the initiator and the type and amount of swelling monomer. The Janus NPs were successfully used as stabilisers in the ab-initio emulsion polymerization to control the particle size and particle size distribution. We have offered a very simple, versatile and environmentally friendly method to produce surfactant free latex polymer(s) particles with decorated/functional surface. 


\section{Acknowledgements}

We acknowledge the support of Dr Algi Serelis of DuluxGroup Australia for preparing the RAFT agent; Dr Meiliana Siauw for the synthesis of the RAFT-Sty $y_{9}-b-\mathrm{AAm}_{15}$ diblock; Dr Chiara Neto and Dr Duc Nguyen for helpful discussion. The authors acknowledge the facilities, and the technical assistance, of the Australian Microscopy \& Microanalysis Research Facility at the Australian Centre for Microscopy and Microanalysis, The University of Sydney. The Key Centre for Polymers and Colloids was established under the Australian Research Council's Research Centres Program.

\section{References}

1 K. K. Zhang, M. Jiang and D. Y. Chen, Self-assembly of particles The regulatory role of particle flexibility, Prog. Polym. Sci., 2012, 37(3), 445-486.

2 J. Z. Du and R. K. O'Reilly, Anisotropic particles with patchy: multicompartment and Janus architectures: preparation and application, Chem. Soc. Rev., 2011, 40(5), 2402-2416.

3 K. J. Lee, J. Yoon and J. Lahann, Recent advances with anisotropic particles, Curr. Opin. Colloid Interface Sci., 2011, 16(3), 195-202.

4 M. Lattuada and T. A. Hatton, Synthesis, properties and applications of Janus nanoparticles, Nano Today, 2011, 6(3), 286-308.

5 V. N. Paunov and O. J. Cayre, Supraparticles and \”Janus\" particles fabricated by replication of particle monolayers at liquid surfaces using a gel trapping technique, Adv. Mater., 2004, 16(9-10), 788-791.

6 A. Perro, et al., Design and synthesis of Janus micro- and nanoparticles, J. Mater. Chem., 2005, 15(35-36), 3745-3760.

7 K.-H. Roh, D. C. Martin and J. Lahann, Biphasic Janus particles with nanoscale anisotropy, Nat. Mater., 2005, 4(10), 759-763.

8 L. Hong, S. Jiang and S. Granick, Simple Method to Produce Janus Colloidal Particles in Large Quantity, Langmuir, 2006, 22(23), 9495-9499.

9 T. Nisisako, et al., Synthesis of monodisperse bicolored Janus particles with electrical anisotropy using a microfluidic co-flow system, Adv. Mater., 2006, 18(9), 1152-1156.

$10 \mathrm{~L}$. Nie, et al., One-pot synthesis of amphiphilic polymeric Janus particles and their self-assembly into supermicelles with a narrow size distribution, Angew. Chem., Int. Ed., 2007, 46(33), 6321-6324.

11 A. Walther, et al., Janus Discs, J. Am. Chem. Soc., 2007, 129(19), 6187-6198.

12 C.-C. Ho, et al., Novel Fabrication of Janus Particles from the Surfaces of Electrospun Polymer Fibers, Langmuir, 2008, 24(11), 5663-5666.

13 S. Jiang, et al., Solvent-Free Synthesis of Janus Colloidal Particles, Langmuir, 2008, 24(18), 10073-10077.

14 A. Walther and A. H. E. Mueller, Janus particles, Soft Matter, 2008, 4(4), 663-668.
15 F. S. Romanski, et al., Production and Characterization of Anisotropic Particles from Biodegradable Materials, Langmuir, 2012, 28(8), 3756-3765.

16 N. Chaturvedi, et al., Simple fabrication of snowman-like colloids, J. Colloid Interface Sci., 2012, 371, 28-33.

17 Y. H. Wang, et al., Emulsion Interfacial Synthesis of Asymmetric Janus Particles, Macromolecules, 2011, 44(10), 37873794 .

18 T. Nisisako and T. Hatsuzawa, A microfluidic cross-flowing emulsion generator for producing biphasic droplets and anisotropically shaped polymer particles, Microfluid. Nanofluid., 2010, 9(2-3), 427-437.

19 T. Tanaka, et al., Preparation of "Mushroom-like" Janus Particles by Site-Selective Surface-Initiated Atom Transfer Radical Polymerization in Aqueous Dispersed Systems, Langmuir, 2010, 26(11), 7843-7847.

20 N. Saito, et al., Formation of "Snowmanlike" polystyrene/ poly(methyl methacrylate)/toluene droplets dispersed in an aqueous solution of a nonionic surfactant at thermodynamic equilibrium, Langmuir, 2007, 23(23), 11506-11512.

21 D. J. Kraft, et al., Patchy Polymer Colloids with Tunable Anisotropy Dimensions, J. Phys. Chem. B, 2011, 115(22), 71757181.

22 H. R. Sheu, M. S. El-Aasser and J. W. Vanderhoff, Phase separation in polystyrene latex interpenetrating polymer networks, J. Appl. Polym. Sci., Part A: Polym. Chem., 1990, 28(3), 22.

23 E. B. Mock, et al., Synthesis of Anisotropic Nanoparticles by Seeded Emulsion Polymerization, Langmuir, 2006, 22(9), 4037-4043.

24 C. Kaewsaneha, et al., Janus Colloidal Particles: Preparation, Properties, and Biomedical Applications, ACS Appl. Mater. Interfaces, 2013, 5(6), 1857-1869.

25 G. Moad, E. Rizzardo and S. H. Thang, Radical additionfragmentation chemistry in polymer synthesis, Polymer, 2008, 49(5), 1079-1131.

26 C. J. Ferguson, et al., Effective Ab Initio Emulsion Polymerization Under RAFT Control, Macromolecules, 2002, 35, 9243-9245.

27 C. J. Ferguson, et al., Ab Initio Emulsion Polymerization by RAFT-Controlled Self-Assembly, Macromolecules, 2005, 38(6), 2191-2204.

28 B. T. T. Pham, et al., Miniemulsion Polymerization Stabilized by Amphipathic Macro RAFT Agents, Macromolecules, 2003, 36(24), 8907-8909.

29 B. T. T. Pham, et al., Miniemulsion Polymerization with Arrested Ostwald Ripening Stabilized by Amphiphilic RAFT Copolymers, Macromolecules, 2010, 43(19), 7950-7957.

30 D. Nguyen, C. Such and B. Hawkett, Polymer-TiO2 Composite Nanorattles via RAFT-Mediated Emulsion Polymerization, J. Polym. Sci., Part A: Polym. Chem., 2012, 50(2), 346-352.

31 D. Nguyen, et al., Pigment Encapsulation by Emulsion Polymerization Using Macro-RAFT Copolymers, Langmuir, 2008, 24(5), 2140-2150.

32 B. Charleux, et al., Polymerization-Induced Self-Assembly: From Soluble Macromolecules to Block Copolymer 
Nano-Objects in One Step, Macromolecules, 2012, 45(17), 6753-6765.

33 M. J. Monteiro and M. F. Cunningham, Polymer Nanoparticles via Living Radical Polymerization in Aqueous Dispersions: Design and Applications, Macromolecules, 2012, 45 (12), 4939-4957.

34 D. Nguyen, C. H. Such and B. S. Hawkett, Polymer coating of carboxylic acid functionalized multiwalled carbon nanotubes via reversible addition-fragmentation chain transfer mediated emulsion polymerization, J. Polym. Sci., Part A: Polym. Chem., 2013, 51(2), 250-257.

35 D. E. Ganeva, et al., Particle Formation in ab Initio RAFT Mediated Emulsion Polymerization Systems, Macromolecules, 2007, 40(17), 6181-6189.

36 A. M. Telford, et al., Micron-sized polystyrene particles by surfactant-free emulsion polymerization in air: Synthesis and mechanism, J. Polym. Sci., Part A: Polym. Chem., 2013, 51(19), 3997-4002. 